\title{
La evaluación de las actividades de Ciencia, Tecnología, Desarrollo e Innovación
}

Evaluation of Activities of Science, Technology,
Development And Innovation
Avaliação das Atividades de Ciência,

Tecnologia, Desenvolvimento e Inovação
Fecha de Recepción

30 de mayo de 2016
Aceptado para su publicación

18 de agosto de 2016
Silvia Matilde Mazza

Profesora Titular. Cátedra Metodología de la Investigación. Facultad de Odontología.UNNE.

E-mail:smmazza@gmail.com.ar

Alina Noelia Peláez

Auxiliar Docente. Cátedra Metodología de la Investigación. Facultad de Odontología. UNNE.

María Natalia Rosende Jefe de Trabajos Prácticos. Cátedra de Psicología del niño, adolescente y el adulto. Facultad de Odontología. UNNE.

Lugar de Trabajo Facultad de Odontología. Universidad Nacional del Nordeste. Avenida Libertad 5450. Teléfono Fax: 03794- 4457992. Corrientes Argentina. C.P. 3400.

\section{Resumen}

La evaluación institucional es un proceso complejo y forma parte del establecimiento de políticas y de la planificación. La evaluación de la Actividad Científica y Tecnológica es reconocida como obligación permanente del Estado y de las Instituciones pertenecientes al Sistema, contribuye al incremento de la calidad y al fortalecimiento dichas Instituciones e implica una serie de definiciones tratando de lograr unanimidad a la hora de considerar términos y métodos para obtener resultados comparables y homogéneos.

Esta evaluación conlleva la necesidad de contar con elementos capaces de determinarla efectividad o eficiencia de la actividad y los posibles usos, con el objetivo de buscar una racionalidad de los recursos de acuerdo con los intereses del desarrollo. La necesidad de medir tanto las Inversiones (humanas y económicas) como de qué manera esas inversiones revierten en la Sociedad, implica que se consideren también indicadores de Producto.

Al definir indicadores se deben considerar grados crecientes de complejidad en los niveles de la evaluación: nacional, institucional, por área disciplinaria o de desarrollo e individual. Por otra parte, se debe atender fundamentalmente a la necesidad de obtener datos comparativos y de definir, de manera homogénea dentro del sistema, con qué medir las diferentes cuestiones. 
REVISTA FACULTAD DE ODONTOLOGÍA

ISSN No 1668-7280 - Vol. IX No 1 - 2016

56

DIVULGACIÓN

Mazza - Peláez - Rosende

Palabras Claves

Evaluación Institucional; Inversiones; Producto.

\section{Abstract}

The institutional evaluation is a complex process and is part of policy-making and planning. Assessment of Scientific and Technological Activity is recognized as a permanent obligation of the State and the institutions belonging to the system it contributes to improving quality and strengthening those institutions and involves a series of definitions trying to achieve unanimity when considering terms and methods to obtain comparable and consistent results.

This assessment involves the need for elements capable of determining it effectiveness or efficiency of the activity and possible uses, with the aim of seeking a rationality of resources in accordance with the interests of development. The need to measure both investments (human and financial) and how these investments reinvested in the company, which also implies product indicators are considered.

When defining indicators should consider increasing degrees of complexity in assessment levels: national, institutional, for disciplinary or developmental and individual area. Furthermore, attention should be paid primarily to the need to obtain comparative data and define homogeneously within the system, how to measure different issues.

\section{Keywords}

Institutional Evaluation; Investments; Product.

\section{Resumo}

A avaliação institucional é um processo complexo e faz parte da formulação de políticas e planejamento. Avaliação da actividade científica e tecnológica é reconhecida como uma obrigação permanente do Estado e as instituições pertencentes ao sistema que contribui para melhorar a qualidade e reforçar as instituições e envolve uma série de definições que tentam obter a unanimidade quando se considera termos e métodos para obter resultados comparáveis e consistentes.
Essa avaliação envolve a necessidade de elementos capazes de determinar que a eficácia ou eficiência da actividade e usos possíveis, com o objetivo de buscar uma racionalidade de recursos de acordo com os interesses do desenvolvimento. A necessidade de medir os investimentos (humanos e financeiros) e como esses investimentos reinvestidos na empresa, o que implica também indicadores de produto são consideradas.

Ao definir indicadores devem considerar o aumento graus de complexidade nos níveis de avaliação: nacionais, institucionais, para a área disciplinar ou de desenvolvimento e individual. Além disso, deve ser dada atenção principalmente para a necessidade de obter dados comparativos e definir de forma homogénea dentro do sistema, como medir questões diferentes.

\section{Palavras chave}

Avaliação Institucional; Investimentos; Produto.

\section{Desarrollo}

La evaluación institucional y las universidades

La evaluación institucional supone un proceso complejo para determinar el valor de algo, que implica la delicada tarea de interpretación de un conjunto de elementos que interactúan configurando una realidad particular y significativa. De hecho, evaluar no es, rigurosamente la etapa posterior o final de un proceso, sino un momento en el camino para apreciar lo caminado, y decidir cómo continuar. Debe ser entendida como parte del proceso de establecimiento de políticas institucionales y de planificación. Si se sigue profundizando, se puede decir que la evaluación que se formula debe aspirar a la credibilidad y al consenso. Una evaluación, asimismo, no es "los datos", aunque éstos sean indispensables, sino el proceso por el que se aprecia y discierne el valor de las acciones y realizaciones; un proceso profundamente humano que se nutre y se articula en el diálogo, la discusión y la reflexión'.

Los fines básicos de las universidades son la adquisición, apropiación y generación de conocimientos; su transmisión, la formación de profesionales e investigadores con sentido crítico, tanto en el nivel de grado como de posgrado; la integración al medio y la contribución a su de- 
sarrollo sustentable y a su bienestar, fundado en los valores de libertad, igualdad, solidaridad y justicia. Para el cumplimiento de estos fines, dichas instituciones cuentan, en diferente medida, con estructuras, funciones procesos de interacción, recursos humanos, materiales y financieros, actividades administrativas y un conjunto normativo enmarcados en un contexto social propio ${ }^{2}$.

La evaluación institucional debe ser útil fundamentalmente a la propia universidad evaluada y a la comunidad en general. Efectivamente, se trata de mejorar la calidad de la institución a través de interrogarse sobre los resultados, y especialmente sobre las acciones, identificando problemas y comprendiéndolos en su contexto. La evaluación institucional debe tender a crear las condiciones óptimas para que los participantes, incluyendo al evaluador, mejoren su comprensión sobre la realidad institucional, como una herramienta para la planificación y la definición de políticas institucionales.

La evaluación de las actividades de ciencia, tecnología, desarrollo e innovación

Planificar consiste en escoger alternativas en situaciones que aún no se han presentado, que son interrelacionadas e interdependientes, y que no son conocidas con certeza. Al comprometerse por adelantado y tomar decisiones en la actualidad, será posible ejercer influencia sobre sucesos futuros. La planificación está dirigida a lograr mayor control sobre estos sucesos futuros, y orientarlos en la dirección apropiada, con el fin de obtener resultados deseados y prever sus posibles consecuencias. La planificación y las decisiones por anticipado que la conforman están destinadas principalmente a generar, identificar y evaluar alternativas. La diferencia entre establecer políticas (policy-making) y planificar (planning) consiste en que establecer políticas involucra fijar criterios para generar e identificar alternativas y elegir entre ellas. Por lo tanto, se puede definir la planificación como la suma de actividades que, sobre la base de los principios y criterios fijados al establecer políticas, generan e identifican alternativas y seleccionan entre ellas mediante un proceso de toma de decisiones por anticipado. Por lo tanto, se puede considerar que establecer políticas es aquella parte del proceso de planificación cuya tarea consiste en fijar principios y criterios para la toma de decisiones por anticipado ${ }^{3}$.

La planificación de la Ciencia y la Técnica en América Latina es una actividad reciente, que no ha podido superar aún múltiples obstáculos y dificultades, como lo demuestran los planes publicados en varios países de la región y que no son sino apenas largas listas de buenas intenciones y retóricos objetivos. Tan pobre resultado se debe, por una parte, a la falta de datos técnicos imprescindibles para formular cualquier plan, como la inexistencia de inventarios de recursos humanos y materiales, la escasez de información sobre las demandas concretas en Ciencia y Técnica por parte del sistema productivo, etc., como así también a la falta de un andamiaje teórico sobre el cual fundamentar la planificación deseada ${ }^{4}$. De lo que se deduce la necesidad de contar con información válida, confiable y fundamentalmente comparable y homogénea sobre la cual basar cualquier intento de planificación.

Por otra parte, la Evaluación de la Actividad Científica y Tecnológica es reconocida como obligación permanente del Estado y de las Instituciones pertenecientes al Sistema. De esa manera lo establecen los artículos 23 y 24 de la Ley Marco 25.467 de Ciencia Tecnología e Innovación de la República Argentina. Entendida en el marco de la Evaluación Institucional, esta tarea involucra tanto a las Universidades como a las otras Instituciones que componen el Sistema Científico Tecnológico.

En ese marco, el "Programa de Evaluación Institucional" (PEI), que funciona bajo el ámbito de la Subsecretaría de Evaluación Institucional, perteneciente a la Secretaría de Articulación Científico Tecnológica del Ministerio de Ciencia, Tecnología e Innovación Productiva, promueve la evaluación permanente y el mejoramiento continuo de las instituciones pertenecientes al Sistema Nacional de Ciencia, Tecnología e Innovación, brindando para ello apoyo técnico y/o económico. Se orienta fundamentalmente a dos tipos de instituciones:

- Los Organismos de Ciencia y Tecnología, en los que la evaluación abarca al organismo en su conjunto. Su diseño tiene un alcance institucional y contempla tanto la misión y objetivos, como los procesos de gestión organizacional y económica, la 
REVISTA FACULTAD DE ODONTOLOGÍA

ISSN No 1668-7280 - Vol. IX No 1 - 2016

58
DIVULGACIÓN

Mazza - Peláez - Rosende actividad científico-tecnológica y sus resultados.

- Las Universidades, en cuyo caso solo se analiza la función Investigación y Desarrollo (I+D) que en ellas se realiza. No son objeto de estudio por parte del PEI las funciones relativas a la gestión organizacional (salvo en el caso de los institutos de investigación dependientes de la universidad, pero descentralizados en lo administrativo) ni la docencia.

No obstante pensar en evaluar la actividad implica una serie de definiciones $y$, desde los inicios, lograr unanimidad a la hora de considerar términos y métodos para obtener resultados comparables y homogéneos. Tradicionalmente, suelen emplearse indicadores de inversión (inputs), de resultados obtenidos (outputs) y de los impactos que esos resultados suponen en determinados ámbitos (outcomes). Los indicadores de inversión tratan de establecer los recursos, tanto económicos como materiales y humanos dedicados a I+D en Ciencia y Tecnología. Estos indicadores permiten comparar la distribución de esos recursos en los distintos países y regiones, además de mostrar su evolución temporal en un determinado dominio geográfico. Las actividades de $I+D$, que a largo plazo contribuyen a la mejora tecnológica, tienen a corto plazo unos resultados mucho más tangibles en forma de publicaciones científicas (además de patentes, creación de spin-offs y otros). Por lo general, para medir y evaluar la cantidad y la calidad de la producción científica se utilizan diversos indicadores bibliométricos. Estas técnicas también pueden ser utilizadas como instrumentos de prospectiva, al permitir la identificación de los campos más innovadores y comprobar la repercusión e interés de los resultados científicos ${ }^{5}$.

Al momento de definir indicadores se deben tener en cuenta grados crecientes de complejidad en los niveles de la evaluación: nacional, institucional, por área disciplinaria o de desarrollo y la evaluación individual. Por otra parte, se debe atender fundamentalmente a la necesidad de obtener datos comparativos, de definir con qué medir diferentes cuestiones como la productividad en I+D (a través de Publicaciones, Patentes, Encuestas de Innovación y considerar otros posibles productos).
Hacia un sistema de indicadores

La OECD (Organizationfor Economic Cooperation and Development), es un foro donde 30 gobiernos democráticos trabajan conjuntamente para afrontar los retos económicos, sociales y medio ambientales de la globalización. La Organización encabeza los esfuerzos destinados a entender y ayudar a los gobiernos a responder a nuevos retos y preocupaciones. Proporciona un marco en el que dichos gobiernos pueden comparar sus experiencias políticas, buscar respuestas a problemas comunes, identificar las "mejores prácticas" y coordinar sus políticas nacionales e internacionales. En 1963 desarrolló una Propuesta de Norma Práctica para Encuestas de Investigación y Desarrollo, que ha llegado a ser más conocido como el "Manual de Frascati". Trata de la medición de los recursos humanos y financieros dedicados a la I+D, a menudo denominados "datos de entrada" (inputs) de la I+D.

Considerando que, para garantizar un seguimiento de la I+D, es esencial disponer de estadísticas e indicadores fiables y comparables, a partir de la quinta edición del Manual se potencian las diversas recomendaciones y directrices metodológicas, especialmente para mejorar las estadísticas de $I+D$, en el sector servicios, así como en la recogida de datos más detallados sobre los recursos humanos en I+D. La publicación 2015 es la séptima edición de un manual, que fue escrito por primera vez en un contexto económico y geopolítico diferente del actual. Por más de 50 años el Manual de Frascati de la OECD ha sido el estándar mundial reconocido para la recopilación y reporte internacional de estadísticas comparables sobre los recursos financieros y humanos dedicados a la investigación y desarrollo experimental. Los esfuerzos coordinados entre los diversos países (tanto dentro como fuera de la OECD) para definir y poner en práctica las recomendaciones de este manual han dado lugar a una valiosa fuente de evidencia para la ciencia, la investigación y fundamentalmente para los responsables de las políticas económicas ${ }^{5}$.

Aunque el Manual es esencialmente un documento técnico, constituye uno de los pilares de las acciones desarrolladas por la OECD para que se comprenda mejor el papel de la Ciencia y la Tecnología mediante el análisis de los sistemas 
nacionales de innovación. Además, al proporcionar definiciones de la I+D, aceptadas internacionalmente, y clasificaciones de sus actividades, el Manual contribuye a los debates intergubernamentales sobre las "mejores prácticas" en materia de políticas científicas y tecnológicas. El Manual de Frascati no es solo una referencia para las encuestas de I+D en los países miembro de la OECD, constituye la norma para las encuestas de I+D en todos los países del mundo ${ }^{5}$.

Investigación y Desarrollo (I+D) se considera cada vez más como una entrada a la innovación en el contexto de los esfuerzos generales realizado en una economía global basada en el conocimiento, pero sigue desempeñando un papel crucial y es un foco importante de las políticas del gobierno, debido a sus características únicas. De acuerdo con la definición contenida en el Manual de Frascati sobre I+D, la actividad "comprende todo el trabajo creativo llevado a cabo sobre una base sistemática en orden de incrementar el stock de conocimiento, incluyendo el conocimiento del hombre, cultura y sociedad, y el uso de esos stocks para idear nuevas aplicaciones"5.

El "Manual de Oslo", producido también por la OECD, brinda Directrices para la Recogida e Interpretación de Información Relativa a Innovación. El Manual de Oslo ha sido elaborado conjuntamente por Eurostat y la OECD y forma parte de la familia de manuales, en continua evolución, dedicados a la interpretación de datos de Ciencia, Tecnología e Innovación. Dicha familia está compuesta por manuales, directrices y guías dedicadas a la I+D (Manual de Frascati), a indicadores de globalización, a la sociedad de la información, a los recursos humanos en ciencia y tecnología (Manual de Canberra) y a estadísticas de biotecnología. Su utilización no se restringe a los países de la OECD, cada vez más países de Latinoamérica, Europa del Este, Asia y África están desarrollando encuestas a partir del Manual de Oslo' .

Aunque, en general, el diseño de estas encuestas partió de los estándares del Manual, muchos de ellos están adaptando la metodología empleada para así recoger las necesidades específicas y las características particulares de sus sistemas estadísticos, dado que poseen estructuras económicas y sociales diferentes a las de los países más desarrollados de la OECD. Las adaptacio- nes se están realizando de manera independiente para cada país $y$, por tanto, con diferentes enfoques. Por ejemplo, la difusión y los cambios incrementales suponen una parte considerable de la innovación que se realiza en los países no pertenecientes a la OECD. La rica y diversa experiencia que estas encuestas han generado ha servido de base para la realización de un anexo en la última edición del Manual, que recoge algunas de las lecciones aprendidas y aporta directrices para encuestas de innovación futuras en los países no pertenecientes a la OECD'.

El Ministerio de Ciencia, Tecnología e Innovación Productiva dela República Argentina (MINСуT) posee un Sistema Integrado de Indicadores de Ciencia, Tecnología e Innovación, que reúne la principal información científica, tecnológica y de innovación generada por la Dirección Nacional de Información Científica. Los indicadores, documentos y publicaciones generados brindan información actualizada para la toma de decisiones en materia de políticas públicas, como así también para el conjunto de la sociedad ${ }^{7}$. La información de base para la construcción de los indicadores surge del operativo denominado "Relevamiento de entidades que realizan actividades científicas y tecnológicas", que se lleva a cabo anualmente desde 1994, cumpliendo con el Decreto $\mathrm{N}^{\circ}$ I.83 I/93. Este operativo obtiene información de las entidades dedicadas a actividades de ciencia y tecnología pertenecientes a distintos ámbitos: organismos públicos, universidades públicas y privadas y entidades sin fines de lucro. Con el propósito de mejorar y robustecer la calidad de la información relevada, desde el año 2010 se ha adoptado una nueva metodología de captura y control de datos. El MINCyT desarrolló una herramienta que permite a los usuarios ingresar los datos solicitados y realizar ciertas verificaciones de consistencia de forma online. No obstante, hasta el año 2013 las publicaciones anuales presentaban algún nivel de detalle de la metodología empleada, en el último número (20l4) solamente se presentan gráficos y tablas con datos sin ninguna información metodológica ${ }^{7}$.

Por otra parte el Consejo Interuniversitario Nacional (CIN) se encuentra trabajando en la definición de un Sistema de Indicadores de la Actividad Científico Tecnológica para ser aplica- 
do en el ámbito de las Universidades Nacionales. Esta tarea, que se desarrolla en articulación con el MINCyT, permitirá contar con información compatible de todo el Sistema Universitario Público de la Nación. No obstante, constituye un desafío que deben afrontar todas las Instituciones del Sistema Científico Tecnológico $\mathrm{Na}$ cional, a la hora de acordar conceptos, criterios y metodología que contribuyan a la comparabilidad de los datos. Otro reto que se presenta es lograr estadísticas ciertas y depuradas, dada muchas veces la doble o triple dependencia de institutos, recursos humanos, tecnológicos y económico-financieros.

\section{Aspectos a tener en cuenta}

Pensar en la evaluación de actividades de I+D involucra una serie de aspectos complejos que pueden resumirse en: a) indicadores que miden Inversiones (humanas y económicas) y b) de qué manera esas inversiones revierten en la Sociedad, para ello se consideran indicadores de Producto, como pueden ser los indicadores de Innovación, de Patentes y los indicadores Bibliométricos ${ }^{8}$.

Al momento de evaluar las actividades de I+D, lo primero que se debe determinar es si realmente constituyen actividades de I+D. Un determinado proyecto puede ser I+D si se realiza por una cierta razón, pero no lo será si se lleva a cabo por otra. De acuerdo con el Manual de Frascati El término I+D engloba tres actividades: investigación básica, investigación aplicada y desarrollo experimental (OECD, 20I5).

Una vez definida que una actividad corresponde a I+D es necesario definir los diferentes indicadores que se van a considerar. Algunos criterios para la definición de Indicadores consideran los indicadores de inversión (inputs) y los de resultados (outputs) y fundamentalmente estos últimos varían en función del tipo de actividad de I+D de que se trata. Una propuesta, desarrollada por Mazza ${ }^{8}$ incluye:

\section{A. Indicadores de Inversión}

Tasa de Gastos en I+D: Gasto total en I+D, en porcentaje sobre el Presupuesto de la Institución.
Tasa de Gastos por Actividad de I+D: Discriminado por actividad financiada (proyectos, salarios de personal de investigación y de apoyo a la investigación, formación de recursos humanos: becarios, formación complementaria).

$\checkmark$ Becarios: $N^{\circ}$ total de becarios de I+D en relación a dedicación de tiempo completo, por categoría.

$\checkmark$ Personal e Investigadores: $N^{\circ}$ total de personas dedicadas a actividades de $I+D$ en relación a dedicación de tiempo completo.

\section{B. Indicadores de Resultados}

\section{Investigación Básica}

a. Producción:

$\checkmark \mathrm{N}^{\circ}$ artículos científicos, discriminados por tipo de revistas (internacionales, nacionales, locales, indexadas, no indexadas, con y sin referato).

$\checkmark \mathrm{N}^{\circ}$ de presentaciones en reuniones científicas y congresos discriminados por alcance de la reunión (local, nacional, internacional) y por especificidad (disciplinar, general).

b. Productividad:

$\checkmark \mathrm{N}^{\circ}$ de artículos científicos por tipo de revista, en relación con los proyectos y en relación con la cantidad de personal de tiempo completo.

$\checkmark \mathrm{N}^{\circ}$ de trabajos presentados en reuniones científicas y congresos, por alcance y especificidad, en relación con los proyectos y en relación con la cantidad de personal de tiempo completo.

$\checkmark \mathrm{N}^{\circ}$ de tesis terminadas ( $\mathrm{N}^{\circ}$ de postgraduados).

$\checkmark$ Tasa de Post-graduación (Relación becarios/ post-graduación).

$\checkmark \mathrm{N}^{\circ}$ de tesinas y trabajos finales de carrera terminados ( $\mathrm{N}^{\circ}$ de graduados).

\section{Investigación Aplicada}

\section{a. Producción:}

$\checkmark \mathrm{N}^{\circ}$ artículos científicos, discriminados por tipo de revistas (internacionales, nacionales, locales, indexadas, no indexadas, con y sin referato).

$\checkmark \mathrm{N}^{\circ}$ de presentaciones en reuniones científicas y congresos discriminados por alcance 
de la reunión (local, nacional, internacional) y por especificidad (disciplinar, general).

$\checkmark N^{\circ}$ de transferencias al sector social o productivo. Indicar los instrumentos de transferencia y las instituciones beneficiarias.

b. Productividad:

$\checkmark \quad N^{\circ}$ de artículos científicos por tipo de revista, en relación con los proyectos y en relación con la cantidad de personal de tiempo completo.

$\checkmark \mathrm{N}^{\circ}$ de trabajos presentados en reuniones científicas y congresos, por alcance y especificidad, en relación con los proyectos y en relación con la cantidad de personal de tiempo completo.

$\checkmark \mathrm{N}^{\circ}$ de tesis terminadas ( $\mathrm{N}^{\circ}$ de postgraduados).

$\checkmark$ Tasa de Post-graduación (Relación becarios/ post-graduación).

$\checkmark \mathrm{N}^{\circ}$ de tesinas y trabajos finales de carrera terminados ( $\mathrm{N}^{\circ}$ de graduados).

$\checkmark \mathrm{N}^{\circ}$ de transferencias, en relación con los proyectos y en relación con la cantidad de personal de tiempo completo.

\section{Desarrollo Tecnológico}

a. Producción:

$\checkmark N^{\circ}$ de desarrollos e innovaciones tecnológicas transferidas.

$\checkmark \mathrm{N}^{\circ}$ de patentes obtenidas.

b. Productividad:

$\checkmark \mathrm{N}^{\circ}$ de desarrollos tecnológicos transferidos, en relación con los proyectos y en relación con la cantidad de personal de tiempo completo.

\section{4) Indicadores de Colaboración}

\section{$\checkmark N^{\circ}$ Publicaciones en colaboración.}

$\checkmark$ Índice de coautoría ( $\mathrm{N}^{\circ}$ medio de autores por producto).

$\checkmark \mathrm{N}^{\circ}$ de instituciones participantes.

$\checkmark \mathrm{N}^{\circ}$ de participantes de otras instituciones.

$\checkmark$ Tasas de colaboración local, regional, nacional e internacional.

\section{5) Indicadores de Distribución}

$\checkmark$ Tasa de Gastos en I+D por área del conocimiento.

$\checkmark$ Tasa de Proyectos de I+D por área del conocimiento. $\checkmark$ Tasa de Becarios por área del conocimiento.

$\checkmark$ Tasa de Investigadores por área del conocimiento.

$\checkmark$ Tasa de productos (artículos discriminados por tipo de revista, presentaciones a congresos y reuniones discriminados por alcance y especificidad, transferencias, desarrollos tecnológicos transferidos, etc.) por área del conocimiento.

$\checkmark$ Tasa de pos graduación por área del conocimiento.

\section{Conclusiones}

Entendida en el marco de la evaluación Institucional, como parte del proceso de establecimiento de políticas y de planificación, la evaluación de las Actividades de Ciencia, Tecnología, Desarrollo e Innovación contribuye al incremento en la calidad y al fortalecimiento de las funciones, tanto de las Universidades como de las otras Instituciones que componen el Sistema Científico Tecnológico.

Realizar una Evaluación Institucional de las Actividades de Ciencia, Tecnología e Innovación implica la necesidad de contar con elementos capaces de evaluar esta actividad desde su efectividad o eficiencia como de sus posibles usos, con el objetivo de buscar una racionalidad de los recursos de acuerdo con los intereses del desarrollo. La necesidad de contar con indicadores que permitan medir las Inversiones (humanas y económicas) y de qué manera esas inversiones revierten en la sociedad, para lo que se consideran indicadores de Producto.

Al momento de definir indicadores también se deben tener en cuenta grados crecientes de complejidad en los niveles de la evaluación: nacional, institucional, por área disciplinaria o de desarrollo y la evaluación individual. Por otra parte, se debe atender fundamentalmente a la necesidad de obtener datos comparativos y de definir de manera homogénea dentro del sistema, con qué medir diferentes cuestiones. 


\section{Bibliografia}

I. Comisión Nacional de Evaluación y Acreditación Universitaria. Lineamientos para la Evaluación Institucional. Buenos Aires. Argentina. 1997.

2. Consejo Interuniversitario Nacional (CIN). Acuerdo Plenario $\mathrm{N}^{\circ}$ 50. Evaluación de la calidad universitaria. Buenos Aires. Argentina. 1992.

3. Sagasti FR. Hacia un nuevo enfoque para la planificación científica y tecnológica. En: Sabato JA. El pensamiento latinoamericano en la problemática ciencia-tecnología-desarrollo-dependencia. Ia ed. Buenos Aires: Ed Biblioteca Nacional.20I I; P 4I5-32.

4. Sabato JA. El pensamiento latinoamericano en la problemática ciencia-tecnología-desarrollodependencia. Ia ed. Buenos Aires: Ed Biblioteca Nacional. 201 I.

5. OECD. Frascati Manual: Guidelines for Collecting and Reporting Data on Research and Experimental Development, The Measurement of Scientific, Technological and Innovation Activities, OECD Publishing, Paris. 2015. [citado 2 de mayo 2016]. Disponible en: http://dx.doi. org/I 0.1787/97892642390I2-en.

6. OECD. Oslo Manual. 3rd Edition. Guidelines for collecting and interpreting innovation data. 2005. [citado 2 de mayo 2016]. Disponible en: www.madrimasd.org

7. Ministerio de Ciencia, tecnología e Innovación Productiva. Indicadores de Ciencia y Tecnología. 2014. [citado 3 de mayo 2016]. Disponible en: http://www.mincyt.gov.ar/indicadores.htm

8. Mazza SM. Indicadores para la Ciencia y la Tecnología. Rev. Gestión Universitaria. 2009; 3 (I) [citado 2 de mayo 2016]. Disponible en: http:// www.gestuniv.com.ar/gu_03/vIn2a2.htm 